\title{
Prevalence of reticular pseudodrusen in an elderly UK Caucasian population-The Bridlington Eye Assessment Project (BEAP): a cross-sectional study (2002-2006)
}

\author{
Craig Wilde ${ }^{1} \cdot$ Ali Poostchi ${ }^{1} \cdot$ Rajnikant L. Mehta ${ }^{2} \cdot$ Jonathan G. Hillman $^{3} \cdot$ Hamish K. MacNab $^{3} \cdot$ Marco Messina $^{1} \cdot$ \\ Marco Morales ${ }^{1} \cdot$ Stephen A. Vernon ${ }^{4} \cdot$ Winfried M. Amoaku (iD ${ }^{1}$
}

Received: 22 June 2017 / Revised: 7 December 2017 / Accepted: 17 December 2017 / Published online: 1 March 2018

(c) The Royal College of Ophthalmologists 2018

\begin{abstract}
Aims: To determine prevalence, associations, and risk factors for reticular pseudodrusen (RPD) in a UK population. Methods: Cross-sectional study of Bridlington residents aged $\geq 65$ years. Masked grading of colour fundus photographs from 3549 participants. RPD presence, phenotype, and topography were recorded, demographic details were analysed, and prevalence was calculated.

Results: RPD was detected in 281 eyes (176 individuals) of 3476 participants (5.06\%) with gradable images, and bilateral in 76.6\%. Digital enhancement increased detection by $15.7 \%$. Prevalence increased significantly with age from $1.18 \%(65-69$ years) to $27.27 \%$ ( $\geq 90$ years) (mean age 81.1 , SD 6.01 ; OR $1.18,95 \%$ CI 1.15-1.21, $p$ value $<0.001$ ), was higher in females (5.9\% vs $4.0 \%$; OR $1.52,95 \%$ CI 1.09-2.13, $p=0.014)$, and associated with diabetes (OR 1.97, CI 1.20-3.17, $p=0.005$ ). History of antihypertension treatment appeared protective (OR $0.64,95 \%$ CI $0.46-0.90, p=0.009)$. RPD subtypes were dot in $18.5 \%$, ribbon in $36.7 \%$, and mixed in $36.3 \%$. RPD were located outside the ETDRS grid in $88 \%$, and most commonly in the outer superior subfield. Central grid involvement occurred in $12.1 \%$ of right and $14.3 \%$ of left eyes. RPD occurred in $25.9 \%$ of participants with grade 4 AMD in at least one eye. RPD was associated with visual dissatisfaction after controlling for age (OR $0.63,95 \%$ CI $0.45-0.88, p=0.007$ ).

Conclusion: RPD occur more commonly than previously reported, most frequently in the upper-outer macular subfield, but also within the central subfield, albeit with reduced frequency and altered morphology. RPD may be associated with visual dissatisfaction and diabetes, but are less frequent in persons receiving antihypertension therapy.
\end{abstract}

Winfried M. Amoaku

Winfried.Amoaku@nottingham.ac.uk

1 Ophthalmology and Vision Sciences, Division of Clinical Neurosciences, B Floor, EENT Centre, Queen's Medical Centre, University of Nottingham, Nottingham, UK

2 Research Design Service, East Midlands (RDS EM), School of Medicine, University of Nottingham, Nottingham Health Science Partners, Room 2107, C Floor South Block, QMC, Nottingham NG7 2UH, UK

3 The Medical Centre, Station Avenue, Bridlington YO16 4LZ, UK

4 University Hospital, Queen's Medical Centre, Nottingham and Honorary Professor of Ophthalmology, University of Nottingham, Nottingham, UK

\section{Introduction}

In 1990, Mimoun et al. [1] described a yellow reticular pattern in the macula of patients with age-related macular degeneration (AMD), initially described as 'pseudodrusen visible on blue light' because of enhanced visibility with blue light fundus photography. In the Wisconsin AgeRelated Maculopathy Grading System (WARMGS), they were classified as soft drusen [2], and not included in the International Classification System of AMD [3]. Arnold et al. [4] subsequently reported the presence of yellow interlacing networks $(125-250 \mu \mathrm{m}$ in width), labelled reticular pseudodrusen (RPD). Based on findings from one histological specimen (without neural retina), they described fibrosis within choroidal stroma, and speculated RPD resulted from poor choroidal perfusion [4]. They suggested that RPD were located below the retinal pigment epithelium (RPE) and increased the risk of AMD [4]. Smith et al. [5] 
correlated RPD on fundus photography (FP) with reticular patterns on fundus autofluorescence (FAF) imaging, and later, investigated RPD with multimodal imaging, including scanning laser ophthalmoscopy (SLO), infrared reflectance (IR) and indocyanine green angiography (ICG). They proposed 'reticular macular disease' (RMD) as a phenotypic entity in the classification of AMD, with the term 'RPD' used to describe en face photographic features [6]. With spectral domain optical coherence tomography (SD-OCT) in 2010, Zweifel et al. [7] localised RPD between RPE and the inner segment/outer segment boundary ('inner segment ellipsoid band' on OCT imaging), contrary to Arnold's histological report. Zweifel et al. [7] referred to them as 'subretinal drusenoid deposits' (SDD). More recently, other investigators have further elucidated their varied localisation in the choroid [8-10].

With multimodal imaging RPD have several clinical morphological features, and variable distribution [11, 12,]. Lee et al. [11] report that RPD range from few discrete deposits localised to the superior macula, to numerous deposits in all four quadrants. Morphology may vary from round to oval, well-defined deposits forming a reticular pattern, to confluent deposits with surrounding branching lesions $[11,13$,]. Diffuse distribution demonstrated a confluent morphological pattern and highest prevalence of late AMD [11].

Despite their importance, there is no population-based report on RPD prevalence in a UK population. To the best of our knowledge, only four population-based studies have reported prevalence and/or incidence of RPD [14-17]. The Beaver Dam Eye Study (BDES) reported a prevalence of $0.7 \%$ at baseline (43-year-old to 86-year-old population), and a 15-year cumulative incidence of $3 \%$ [14]. The Melbourne Collaborative Cohort Study (MCCS) reported a prevalence of $0.4 \%$ [15]; the Blue Mountains Eye Study (BMES) had a 5-year incidence of 2\% [18], and 15-year cumulative incidence as $4.0 \%$ [16]. The Rotterdam Study recently reported a prevalence of $4.9 \%$ in their population aged $\geq 65$ years [17]. Furthermore, none of these studies used digital enhancement of photographs.

This manuscript reports the epidemiology of RPD in an elderly UK population using digital enhancement of CFP images and explores associated risk factors for RPD. We report topographical distribution of RPD along with prevalence rates of different morphological types of RPD as seen with CFP, which to date remains unreported from a cross-sectional population-based study.

\section{Methods}

The BEAP Study methodology, including image acquisition and analysis are described elsewhere [19]. The study received approval from the local ethics committee
(Scarborough and North East Yorkshire Local Ethics Research Committee; Ref No. PB/RH/02/288), and its methodology adhered to the tenets of the Declaration of Helsinki. Image grading was performed in accordance with the International Classification System of AMD [3]. An Early Treatment Diabetic Retinopathy Study (ETDRS) grid was superimposed onto digital images, centred at the fovea, dividing the macula into 9 subfields, consisting of a central subfield, an inner circle (4 inner subfields), and an outer circle (4 outer subfields).

The CFP was initially graded without digital alteration and subsequently with adjustment to the red-free and blue channels of the photograph using the IMAGEnet 2000 program. To do this within the viewing module, 'Utilities' was selected and, out of the three principal colour channels, the red component was minimised. The enhanced image was compared to the first. All images within the BEAP database were analysed as such, even if initial review did not identify RPD.

Each eye was assessed specifically for RPD, which were considered present with a definite reticular pattern of round/ oval yellow-white lesions that joined to form an ill-defined network of broad, interlacing ribbons. A diagnosis was made if there were round/oval lesions, with no interlacing or confluent pattern evident, if lesions were regularly spaced forming a reticular pattern, with significant uniformity in size and colour, and were more clearly visible with enhancement of the digital photograph (to the red-free channel). Only if the grading ophthalmologist was definite that these lesions were not regularly arranged conventional drusen was this grade of RPD assigned.

All questionable RPD lesions were scrutinised by a retinal specialist with expertise in image grading (W.M.A.). Any differences in opinion were sent to the Central Angiographic Reading Facility, Belfast for adjudication. Frequent sessions of simultaneous grading were performed to maintain reproducibility. The presence of RPD was also recorded outside the grid and nasal to the optic disc.

All eyes with RPD were re-evaluated and the presence of RPD was confirmed. Topographical RPD distribution across nine subfields was recorded. Eye with RPD were assessed for conventional drusen, hyperpigmentation, and hypopigmentation within the ETDRS grid on a subfield by subfield basis. All RPD eyes were sub-categorised for the morphological characteristics of RPD as described by Suzuki et al. [12]. Dot RPD were defined as discrete dots, often paler or 'whiter' in colour than conventional drusen, and may be confluent [12]. The lesions had to form a regular pattern. Ribbon RPD was assigned if lesions formed interlocking ribbons that could be confluent. If both RPD types were present, either superimposed on each other within the same area of the fundus, or distributed in separate areas, then a 'mixed RPD' phenotype was recorded [12]. 
After independent grading of the images, LogMAR visual acuities, corrected with both current glasses and pinhole (Baylie Lovie no. 4 chart) for each eye, and participant satisfaction with their vision at the time were merged with the grading.

Statistical analysis was performed using Stata 12.0 (StataCorp, College Station, TX, USA) and SPSS v.22 (IBM Corp. Armonk, NY, USA). Differences in proportions were assessed using Chi-squared tests. Binary outcome variables were analysed using logistic regression techniques. All the statistical tests were two-sided at the 5\% significance level and effect size accompanied with $95 \%$ confidence interval $(95 \% \mathrm{CI})$.

\section{Results}

In total, 3476 Caucasian participants had gradable photographs in at least one eye, and 3255 in both eyes. RPD was present in 281 eyes of 176 individuals $(5.06 \%$ prevalence for either eye), in the $\geq 65$ year age group. Out of the 281 eyes, RPD were only detectable after digital enhancement of the CFP to the red-free channel in $44(15.7 \%)$. RPD frequency was almost identical for right $(n=140,4.18 \%)$ and left $(n=141,4.16 \%)$ eyes. Mean age for subjects with RPD was 81.1 years (SD 6.01). Amongst subjects with gradable photographs in both eyes, 105 (76.6\%) had bilateral RPD. A total of 114 subjects with RPD were female $(64.8 \%)$. Females had statistically significant higher genderspecific prevalence when compared to males (5.9 vs $4.0 \%$, $p=0.014$; Table 1). Prevalence of RPD increased with age, from $1.18 \%$ in the 65-69 year age group, reaching a maximum of $27.27 \%$ in those $\geq 90$ years. Age-specific RPD prevalence is shown in Table 2. RPD was ribbon type in $103(36.7 \%)$, dot in $52(18.5 \%)$, and mixed ribbon and dot in $102(36.3 \%)$ eyes.

Using a logistic regression model with RPD as a dependent variable, age was associated with RPD where a unit increase in age had an $18 \%$ excess risk (OR 1.18, 95\% CI 1.15-1.21, $p<0.001)$. Female gender, compared to male gender had 52\% excess risk (OR 1.52, 95\% CI 1.09-2.13, $p$ $=0.014)$. A history of diabetes mellitus (DM) had $97 \%$ excess risk (OR 1.97, CI 1.20-3.17, $p=0.005$ ). A history of treatment for hypertension had a reduced risk of RPD

Table 1 Gender-specific prevalence of reticular pseudodrusen

\begin{tabular}{llll}
\hline Gender & RPD absent & RPD present & Total \\
\hline Female & $1826(94.12)$ & $114(5.88)$ & $1940(100)$ \\
Male & $1474(95.96)$ & $62(4.04)$ & $1536(100)$ \\
Total & $3300(94.94)$ & $176(5.06)$ & $3476(100)$ \\
\hline
\end{tabular}

Individuals with only one eye/image available have been excluded from the analysis. Data are represented as number (percentage)
(OR $0.64,95 \%$ CI $0.46-0.90, p=0.009$ ). A history of cerebrovascular accident was not associated with RPD (OR 1.00 , CI $0.59-1.69, p=1.00)$. No data on statin use was available and association with RPD was not assessed. RPD had a high prevalence in eyes with advanced AMD (Table 3).

When present, RPD were located outside the ETDRS grid 88.2 and $88.5 \%$ of the time, in right and left eyes, respectively. When within the grid, they were most commonly found in the outer superior subfield, 91.9 and $87.8 \%$, for the right and left eyes, respectively. RPD were found in the central grid in only 12.1 and $14.3 \%$ of right and left eyes. Subjectively, the fundus appearance in many eyes with RPD was tessellated, frequently showing features suggestive of age-related choroidal atrophy (Fig. 1a). Patterns and appearances of RPD were heterogeneous, appearing to change according to region of macula involved. On occasions, as RPD encroached the central subfields dot RPD was more obvious. Closer to the fovea, the lesions were often subtle and smaller. RPD involving the central subfield when present often had a distinct appearance, as shown in Fig. 1b which the authors referred to as 'ultrafine RPD'. The appearance is of an exaggerated central stippling that may form part of a continuum of a central stippled pigmented epitheliopathy often seen in RPD with a foveal sparring geographic atrophy (GA; Fig. 1c). The topographic distribution of RPD is shown in Fig. 1d, e.

The frequency of co-morbid 'conventional' drusen $>125$ $\mu \mathrm{m}$, hyperpigmentary and hypopigmentary RPE changes, and advanced AMD for right and left eyes is shown in Table 3. Approximately $50 \%$ of eyes had conventional drusen greater than $125 \mu \mathrm{m}$. Among the total of 158 subjects with grade 4 AMD in 1 or both eyes, 41 subjects (25.9\%) had RPD in at least 1 eye, with Grade 4c eyes having the highest prevalence. Only 36 (12.8\%) of the 281 eyes with RPD had no or minimal evidence of conventional drusen or pigmentary changes within the ETDRS grid.

The relationship between subject satisfaction with vision and RPD was explored. When controlling for age, there appeared to be an association of visual dissatisfaction with the presence of RPD (OR estimate 0.63 , 95\% CI 0.45-0.88, $p=0.007)$.

\section{Discussion}

The prevalence of RPD in the Bridlington (UK) population (aged $>65$ years) of $5.06 \%$ is similar to the $4.9 \%$ for participants aged $>65$ years recently reported in the Rotterdam Study [17], representing the highest population-based prevalence reported, and is significantly higher than the $0.7 \%$ baseline prevalence (subjects aged 43-86 years) and 3\% 15year cumulative incidence reported by Klein et al. in the 
Table 2 Age-specific prevalence of RPD

\begin{tabular}{llllllll}
\hline RPD & $65-69$ & $70-74$ & $75-80$ & $80-84$ & $85-90$ & 90 and over & Total \\
\hline No & $838(98.82)$ & $1050(98.22)$ & $766(94.80)$ & $479(89.70)$ & $143(77.72)$ & $24(72.73)$ & $3300(94.94)$ \\
& {$[97.81-99.39]$} & {$[97.22-98.88]$} & {$[93.03-96.15]$} & {$[86.82-92.02]$} & {$[71.15-83.15]$} & {$[55.61-85.10]$} \\
Yes & $10(1.18)$ & $19(1.78)$ & $42(5.20)$ & $55(10.30)$ & $41(22.28)$ & $9(27.27)$ & $176(5.06)$ \\
& {$[0.61-2.19]$} & {$[1.12-2.78]$} & {$[3.85-6.97]$} & {$[7.98-13.18]$} & {$[16.85-28.85]$} & {$[14.9-44.39]$} & {$[4.38-5.84]$} \\
Total & $848(100)$ & $1069(100)$ & $808(100)$ & $534(100)$ & $184(100)$ & $33(100)$ & $3476(100)$ \\
\hline
\end{tabular}

Data are represented as number (percentage). [95\% CI]

Table 3 Comorbidity of RPD with conventional drusen, RPE pigmentary changes and AMD

\begin{tabular}{|c|c|c|}
\hline & Right Eye & Left eye \\
\hline \multicolumn{3}{|l|}{ Present within ETDRS grid } \\
\hline Conventional drusen over $125 \mu \mathrm{m}$ & $69(50.37)$ & $74(53.24)$ \\
\hline RPE hyperpigmentation & $10(7.41)$ & $16(11.51)$ \\
\hline RPE hypopigmentation & $2(1.48)$ & $4(2.88)$ \\
\hline Both hyper/hypopigmentation & $28(20.74)$ & $34(24.46)$ \\
\hline Geographic atrophy(AMD Grade $4 a$ ) & $10(15.15)$ & $15(23.81)$ \\
\hline Neovascular AMD(AMD Grade 4b) & $7(18.42)$ & $7(17.50)$ \\
\hline $\begin{array}{l}\text { Peripapillary choroidal neovascular } \\
\text { membrane (AMD Grade 4c) }\end{array}$ & $2(40.00)$ & $1(25.00)$ \\
\hline
\end{tabular}

Data are represented as number (percentage)

BDES. It is considerably higher than the $0.41 \%$ prevalence in the MCCS, (participants aged 48-86 years) [15]. A prevalence of $13.4 \%$ was reported amongst 494 participants aged 77-92 years, increasing to $50 \%$ in participants aged $\geq 85$ years, with multimodal imaging in the Alienor Study [20]. The difference may be explained by the older age group in our population, and the utilisation and analysis of the red-free channel of the CFP, which enhanced RPD detection. The Rotterdam Study [17] utilised near infrared (NIR) imaging, and reported bilateral RPD in 69.3\% compared to $76.6 \%$ in the present study. Similarly, Wu et al. [21] confirmed that NIR and SD-OCT were better at detecting RPD than CFP in their hospital cohort. To the best of our knowledge, no other population-based study has used red-free enhancement of CFP images.

A major criticism of this study is its sole use of CFP, rather than multimodal imaging for RPD detection. Although some other milestone studies have used only CFP, the diagnostic ability of this modality is considered unsatisfactory by some secondary to its low sensitivity [22]. However, its specificity is extremely high (almost 100\%) and therefore it is a powerful confirmatory test [22]. It has been suggested as there is no diagnostic gold standard, at least two imaging modalities should be used [23], with initial screening being undertaken with highly sensitive imaging modalities, such as SD-OCT, in combination with a highly specific one, such as CFP for confirmation [22].
Future RPD/AMD studies should use a multimodal approach for identification.

Previous population studies, like ours, may have underestimated RPD prevalence because of the sole utilisation of standard CFP. Detection of RPD and differentiation from conventional drusen is dependent on recognition of particular fundus features, involving a degree of pattern recognition. There is a potential for significant under-reporting among inexperienced graders and ophthalmologists unfamiliar with the features of RPD. This is more difficult in eyes with co-morbid drusen or pigmentary changes. In this study, the primary grader was an ophthalmologist, with experience in identifying features of RPD using CFP and multimodal modalities. This, and improved awareness/ recognition of the distinctive phenotype, may have contributed to enhanced detection and higher recorded prevalence. Studies show several imaging modalities have greater sensitivity for RPD detection, when compared to CFP. Smith et al. [6] and Ueda-Arakawa et al. [22] compared imaging methods for diagnosing RPD. Both studies demonstrated IR imaging to be the most sensitive modality ( $95 \%$ ) compared to $88 \%$ for CFP [6, 22,]. However, IR has low specificity when compared to other tests $(92 \%)$, possibly because soft drusen have similar appearance on IR [22].

RPD prevalence increases with age $[14,22,24,25$,$] . In$ the MCCS, the highest prevalence was $2.78 \%$ (women aged $\geq 80$ years) [15]. In the Rotterdam Study, there was a steep rise in the occurrence of RPD in persons aged $>90$ years to $\sim 10 \%$ with CFP [17]. In this study, prevalence reached significantly higher levels $(27.3 \%$ in persons aged $>90$ years). The availability of gradable photos for 751 subjects aged $>80$ years, allowed good estimation of RPD prevalence in the oldest individuals, in comparison to the BDES where the oldest participant at baseline was 86 years.

RPD are consistently reported as being more prevalent in females, as supported by results from this study. Arnold et al. [4] reported $87 \%$ of RPD were in woman; Smith et al. [6] reported a similar $79 \%$ female preponderance. Klein et al. [14], after controlling for age, reported a $2 \frac{1}{2} 2$-fold increase in prevalence and incidence in woman Similarly, the Rotterdam Study reported a 2-fold increase in RPD in females compared to males [17]. This disproportionate 


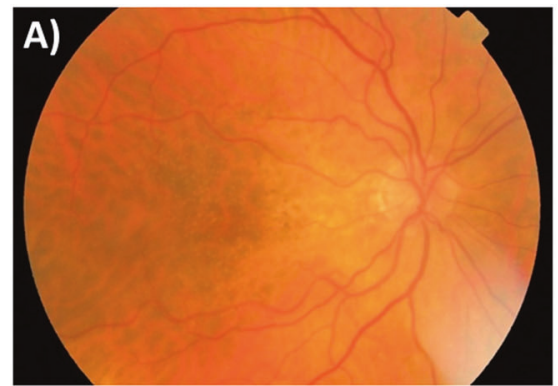

D)

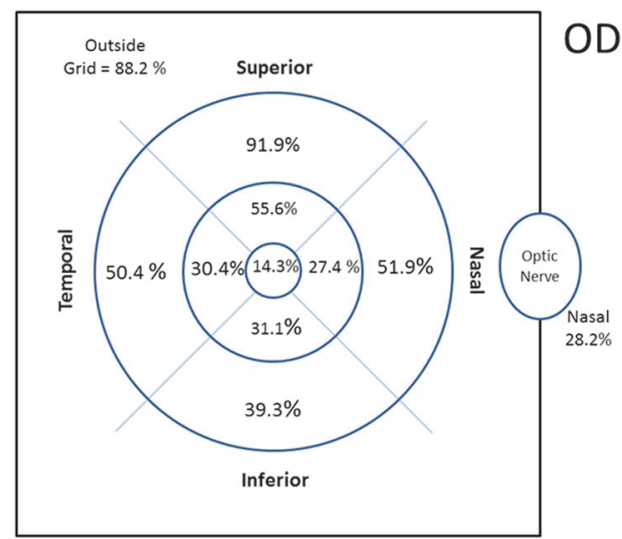

Fig. 1 a Colour fundus photograph illustrating dot reticular pseudodrusen. There are multiple round, generally well defined, equally sized and regularly spaced white lesions located predominantly in the supero-temporal macula area. The fundus is noted to have a tessellated appearance. There is significant temporal peripapillary hypopigmentation, with a prominent area of scleral show. On the temporal border of this area are clusters of RPE hyperpigmentation. b The corresponding left eye of a. There is noticeable symmetry in the tessellated pattern of RPE pigmentation and the distribution of the peripapillary hypopigmentation and scleral show. The dot reticular pseudodrusen follow an almost symmetrical distribution between the two eyes. In the perifoveal area RPD become fainter, with a lower density and smaller diameter. c Subtle dot reticular pseudodrusen are

occurrence in females has not been adequately explained. One suggestion is that RPD may be of autoimmune origin [14], because of the higher prevalence in females, as supported by the association with a history of steroid eye drop use in the BDES [14]. An alternative hypothesis is that as RPD are associated with $54 \%$ decreased survival rate in subjects at baseline in BDES, there may be a systemic cause for RPD that confers increased mortality in males at a younger age. This hypothesis is supported by the increasing evidence of an association between RPD and cardiovascular disease risk factors, such as hypertension [26] and angina [27]. A recent publication demonstrated an association of diffuse-trickling GA (which is strongly associated with RPD) and cardiovascular disease, particularly in males [28], demonstrating that in the $<65$ years age group, $54 \%$ of patients had been admitted to hospital with cardiovascular disease, including hypertensive crisis, angina, and myocardial infarction.

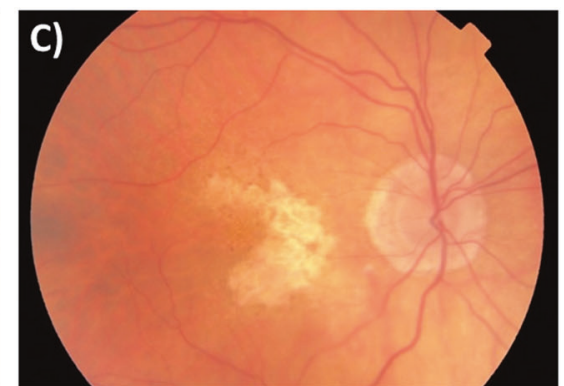

E)

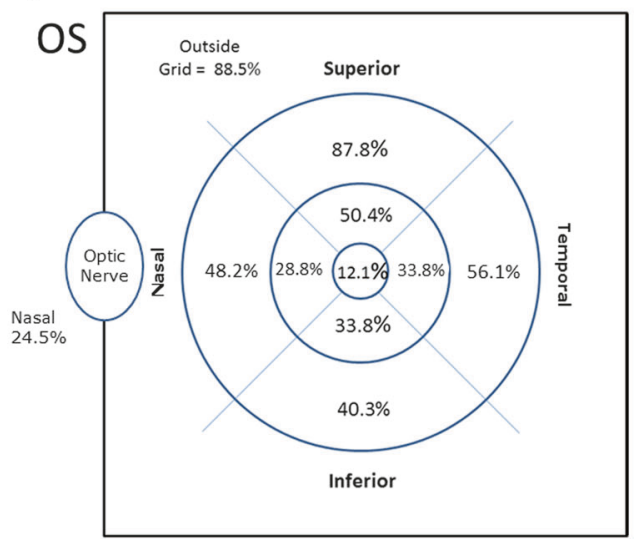

particularly difficult to visualise when there is marked RPE and choroidal hypopigmentation. Colour fundus photographic features of age-related choroidal atrophy are visible, with a tesselatted RPE pattern temporal to the macula, but peripapillary atrophy and scleral show temporal to the disc area. The area of geographic atrophy (GA) is noted to be u-shaped, bright white, and extending into areas where pseudodrusen are most frequent and dense (supero-temporal and inferotemporal macula). Choroidal vessels appear white and sparse within the GA area which is bordered by areas of hyperpigmentation. There is a stippled appearance in the central area. d, e Topographical distribution of reticular pseudodrusen in right and left eyes, respectively

The current study adds further weight to potential associations with cardiovascular risks, demonstrating a positive association with a history of DM. The suggestion of a possible protective effect from hypertension treatment is interesting but putative. It is acknowledged that it may be a statistical anomaly secondary to the small numbers of participants involved. Boddu et al. [28] reports patients with RPD are more likely to be hypertensive than those with large soft drusen. In the MCCS, Finger et al. [15] found that RPD were associated with moderately elevated systolic blood pressure, current smoking and a trend for an association with a history of myocardial infarction or stroke. This effect was reported to disappear with multivariate analysis, indicating a modest effect size. In the BDES, while controlling for age, RPD were associated with, lower income (OR per lower income group 1.75, 95\% CI 1.16, $2.62, p=0.007$ ), body mass index (OR per $1 \mathrm{~kg} / \mathrm{m}^{2} 1.08$, $95 \%$ CI $1.02,1.15, p=0.006)$, and more pack years 
smoked (OR for 35 or more pack years smoked vs none $=$ $2.61,95 \%$ CI $1.17,5.85, p=0.02$ ) [14]. In the BMES, Tan et al. found that high-density lipoproteins (HDL) were inversely related to incidence of late AMD [29]. A history of any cardiovascular disease, including stroke, myocardial infarction, or angina was also associated with incident early AMD and incident soft or reticular drusen [29]. To the best of our knowledge, any suggestion of the possible protective effect of hypertension treatment in relation to RPD has not been reported previously, and clearly warrants further investigation and additional large-targeted studies. Assessment of the impact of different classes of antihypertensive medication, duration of medication use, and the influence of magnitude of blood pressure reduction on risk of RPD development and progression to AMD would offer interesting further studies.

To the best of our knowledge, the association of RPD with visual dissatisfaction when adjusted for age is a new, previously unreported finding. It has previously been demonstrated that RPD decrease retinal sensitivity within the macula irrespective of visual acuity [30]. With microperimetry, Querques et al. (2014) [30] found a difference in the overall macular function between patients with RPD and those with soft drusen only. This is not surprising given the association of RPD with AMD. Dissatisfaction may be explained by photoreceptor dysfunction in the presence of RPD. Alternatively, eyes with RPD and associated choroidal thinning may form part of a spectrum of chorioretinal changes seen in age-related choroidal atrophy (ACA), which is associated with mild vision reduction [31]. This retinal phenotype described by Spaide (2009) [31], including tessellated fundus, peripapillary atrophic changes, rarefaction of choroidal vessels in the macula and central pigmentary changes were sometimes identified in eyes with RPD. However, given the subjective nature of grading such changes on CFP, especially in the absence of enhanced depth imaging to allow choroidal thickness measurement, no association has been objectively explored.

Foveal sparring by RPD is a well-recognised phenomenon [13, 29, 30, [32]]. However, several studies with multimodal imaging techniques have reported central subfield involvement, albeit at lower rates. With confocal SLO, Steinberg reported central subfield involvement in $46 \%$, significantly lower than the surrounding subfields involvement (62-100\%) [33]. Smith et al. [6] similarly demonstrated RPD localisation within the central macula with IR, suggesting that the blue light employed in other modalities is suppressed by luteal pigments, accounting for the apparent absence of reticular lesions in the central zone. In this study, the significantly lower occurrence of RPD in the central subfield than surrounding subfields further supports the concept of relative foveal sparring from RPD, and confirms the predilection of RPD for the superior macula. It is recognised that RPD in the central subfield (on CFP) may have a distinct appearance referred to in this manuscript as 'ultrafine RPD', that may form part of a continuum of a central stippled pigment epitheliopathy often seen in association with RPD in foveal sparring GA or in patients with ACA. Multimodal imaging, particularly including SD-OCT, would have helped differentiate small central RPD from conventional hard drusen with confidence.

To the best of our knowledge, there are no previous reports on the prevalence of RPD subtypes from a population study, and only limited literature is available from hospital-based populations $[11,12]$. This is partly explained by a lack of consensus regarding RPD multimodal imaging and sub-classification. Suzuki et al. [12]. reported dot RPD as the most common subtype, found in $96.1 \%$. This present study confirms that dot RPD are frequent in isolation or mixed with ribbon as identified in $54.8 \%$ of RPD eyes. The lower prevalence in the present study may reflect the different study population (community based cross-sectional study) vs. a hospital-based retrospective review of patients with known RPD [12], where RPD is more obvious. An alternative explanation is the different diagnostic imaging tools utilised. In this study, only digitally enhanced CFP was used and identified ribbon RPD as the most frequent type. Both CFP and IR-SLO were used by Suzuki et al. [12] who specifically reported that dot RPD were most commonly identified using IR-SLO, while ribbon RPD was more detectable using CFP. Our findings are in agreement with the Suzuki study [12]. This study further demonstrates that different subtypes of RPD can occur together or independently of each other. It is reasonable to assume that one type is not a more advanced form of RPD development. It remains to be determined if dot or ribbon RPD confer different risks for development of either GA or neovascular AMD (nAMD).

In agreement with previous reports, this study confirms that RPD are highly associated with advanced AMD. Smith et al. [6] reported that $74 \%$ of subjects with RPD had late AMD, with nAMD being the predominant subtype. In the MCCS, Finger et al. [15] report GA to have the strongest association with RPD. The reasons behind these differences may reflect the tendency for RPD to regress with incident GA or the development of nAMD. Given the predilection for RPD within the supero-temporal arcade, it is likely that RPD would be more easily identified in eyes with a Grade 4c AMD, as the macular area is frequently preserved.

The main limitation of our study is the lack of genetic data, in addition to the lack of multimodal imaging previously highlighted. Previous studies had reported significant associations with $A R M S 2$ and $C F H$ genes [17, 21].

In conclusion, this study demonstrates that RPD are more common than previously reported in population-based studies, with prevalence approximating $5 \%$ in persons aged 
$>65$ years, with a female preponderance. Prevalence increased significantly with age, to a maximum of $27 \%$ in persons aged $>90$ years. It confirms that RPD most frequently occur in the upper-outer macular subfield, and, contrary to previous reports, occur within the central subfield, although less frequently. They are commonly found in association with other signs of ARM, including drusen over $125 \mu \mathrm{m}$ and pigmentary changes. Isolated RPD, in the absence of conventional drusen, is uncommon but does occur. Approximately 1 in 4 subjects with advanced AMD will have evidence of RPD in either eye. RPD appear to be associated with DM. The suggestion that treatment for hypertension may have a protective effect needs further investigation. There is a suggestion that RPD are associated with visual dissatisfaction. However, this will also require further exploration.

\section{Summary}

\section{What was known before}

- RPD are an infrequent finding when compared to conventional drusen, but are strongly associated with AMD.

- RPD have a female preponderance and occur most frequently in a super-temporal fundus distribution.

- They increase in prevalence with age.

\section{What this study adds}

- This is the first UK population-based study to report prevalence of RPD.

- It is the first population-based study to report on the prevalence of RPD with the additional use of digital enhancement of CFP, to enhance case detection.

- RPD occurs in the central subfield, but at a reduced frequency and with altered size/morphology.

- Treatment for hypertension appeared to protect against RPD development while a history of DM may increase risk, but further study is warranted to confirm these findings.

- We demonstrate that with CFP, ribbon RPD are the most prevalent morphological subtype but that different subtypes may occur together or independently of each other. It is, therefore, reasonable to assume that one type is not a more advanced stage of RPD development than the other.

- Within this population the presence of RPD is associated with visual dissatisfaction.

Acknowledgements This research was funded in part by a Research Grant from the Macular Society UK, Andover, Hants, UK. The Bridlington Eye Assessment Project was funded by an unrestricted grant from Pfizer. We would also like to thank the following organisations for financial support of the Project: Pharmacia, Yorkshire Wolds and Coast Primary Care Trust, The Lords Feoffees of Bridlington, Bridlington Hospital League of Friends, The Hull and East Riding Charitable Trust, The National Eye Research Centre (Yorkshire), The Rotary Club of Bridlington, The Alexander Pigott Wernher Memorial Trust, Bridlington Lions Club, The Inner Wheel Club of Bridlington, Soroptimist International of Bridlington, and The Patricia and Donald Shepherd Charitable Trust.

\section{Compliance with ethical standards}

Conflict of interest The authors declare that they have no conflict of interest.

\section{References}

1. Mimoun G, Soubrane G, Coscas G. [Macular drusen]. J Fr Ophtalmol. 1990;13:511-30.

2. Klein R, Davis MD, Magli YL, Segal P, Klein BE, Hubbard L. The Wisconsin age-related maculopathy grading system. Ophthalmology. 1991;98:1128-34.

3. Bird AC, Bressler NM, Bressler SB, Chisholm IH, Coscas G, Davis MD, et al. An international classification and grading system for age-related maculopathy and age-related macular degeneration. The International ARM Epidemiological Study Group. Surv Ophthalmol. 1995;39:367-74.

4. Arnold JJ, Sarks SH, Killingsworth MC, Sarks JP. Reticular pseudodrusen. A risk factor in age-related maculopathy. Retina. 1995;15:183-91.

5. Smith RT, Chan JK, Busuoic M, Sivagnanavel V, Bird AC, Chong NV. Autofluorescence characteristics of early, atrophic, and high-risk fellow eyes in age-related macular degeneration. Invest Ophthalmol Vis Sci. 2006;47:5495-504.

6. Smith RT, Sohrab MA, Busuioc M, Barile G. Reticular macular disease. Am J Ophthalmol. 2009;148:733-43 e732.

7. Zweifel SA, Spaide RF, Curcio CA, Malek G, Imamura Y. Reticular pseudodrusen are subretinal drusenoid deposits. Ophthalmology. 2010;117:303-12.e1.

8. Sohrab MA, Smith RT, Salehi-Had H, Sadda SR, Fawzi AA. Image registration and multimodal imaging of reticular pseudodrusen. Invest Ophthalmol Vis Sci. 2011;52:5743-8.

9. Querques G, Querques L, Forte R, Massamba N, Coscas F, Souied EH. Choroidal changes associated with reticular pseudodrusen. Invest Ophthalmol Vis Sci. 2012;53:1258-63.

10. Alten F, Clemens CR, Heiduschka P, Eter N. Localized reticular pseudodrusen and their topographic relation to choroidal watershed zones and changes in choroidal volumes. Invest Ophthalmol Vis Sci. 2013;54:3250-7.

11. Lee MY, Yoon J, Ham DI. Clinical features of reticular pseudodrusen according to the fundus distribution. Br J Ophthalmol. 2012;96:1222-6.

12. Suzuki M, Sato T, Spaide RF. Pseudodrusen subtypes as delineated by multimodal imaging of the fundus. Am J Ophthalmol. 2014; 157:1005-12.

13. Sarks J, Arnold J, Ho IV, Sarks S, Killingsworth M. Evolution of reticular pseudodrusen. Br J Ophthalmol. 2011;95:979-85.

14. Klein R, Meuer SM, Knudtson MD, Iyengar SK, Klein BE. The epidemiology of retinal reticular drusen. Am J Ophthalmol. 2008;145:317-26.

15. Finger RP, Chong E, McGuinness MB, Robman LD, Aung KZ, Giles G, et al. Reticular pseudodrusen and their association with age-related macular degeneration: The Melbourne Collaborative Cohort Study. Ophthalmology. 2015;123:599-608. 
16. Joachim N, Mitchell P, Rochtchina E, Tan AG, Wang JJ. Incidence and progression of reticular drusen in age-related macular degeneration: findings from an older Australian cohort. Ophthalmology. 2014;121:917-25.

17. Buitendijk GH, Hooghart AJ, Brussee C, de Jong PT, Hofman A, Vingerling JR, et al. Epidemiology of reticular pseudodrusen in age-related macular degeneration: The Rotterdam Study. Invest Ophthalmol Vis Sci. 2016;57:5593-601.

18. Mitchell P, Wang JJ, Foran S, Smith W. Five-year incidence of age-related maculopathy lesions: The Blue Mountains Eye Study. Ophthalmology. 2002;109:1092-7.

19. Wilde C, Poostchi A, Mehta RL, MacNab HK, Hillman JG, Vernon SA, et al. Prevalence of age-related macular degeneration in an elderly UK Caucasian population-The Bridlington Eye Assessment Project: a cross-sectional study. Eye. 2017;31:1042-50.

20. Chan H, Cougnard-Gregoire A, Delyfer MN, Combillet F, Rougier MB, Schweitzer C, et al. Multimodal imaging of reticular pseudodrusen in a population-based setting: The Alienor Study. Invest Ophthalmol Vis Sci. 2016;57:3058-65.

21. Wu Z, Ayton LN, Luu CD, Baird PN, Guymer RH. Reticular pseudodrusen in intermediate age-related macular degeneration: prevalence, detection, clinical, environmental, and genetic associations. Invest Ophthalmol Vis Sci. 2016;57:1310-6.

22. Ueda-Arakawa N, Ooto S, Tsujikawa A, Yamashiro K, Oishi A, Yoshimura N. Sensitivity and specificity of detecting reticular pseudodrusen in multimodal imaging in Japanese patients. Retina. 2013; 33: 490-7.

23. Alten F, Eter N. Current knowledge on reticular pseudodrusen in age-related macular degeneration. $\mathrm{Br} \mathrm{J}$ Ophthalmol. 2015;99:717-22.

24. Puche N, Blanco-Garavito R, Richard F, Leveziel N, Zerbib J, Tilleul J, et al. Genetic and environmental factors associated with reticular pseudodrusen in age-related macular degeneration. Retina. 2013;33:998-1004.

25. Lee MY, Yoon J, Ham DI. Clinical characteristics of reticular pseudodrusen in Korean patients. Am J Ophthalmol. 2012;153:530-5.

26. Boddu S, Lee MD, Marsiglia M, Marmor M, Freund KB, Smith RT. Risk factors associated with reticular pseudodrusen versus large soft drusen. Am J Ophthalmol. 2014;157:985-93 e982.

27. Smith RT, Merriam JE, Sohrab MA, Pumariega NM, Barile G, Blonska AM, et al. Complement factor $\mathrm{H} 402 \mathrm{H}$ variant and reticular macular disease. Arch Ophthalmol. 2011;129:1061-6.

28. Fleckenstein M, Schmitz-Valckenberg S, Lindner M, Bezatis A, Becker E, Fimmers R, et al. The "diffuse-trickling" fundus autofluorescence phenotype in geographic atrophy. Invest Ophthalmol Vis Sci. 2014;55:2911-20.

29. Tan JSL, Mitchell P, Kifley A, Flood V, Smith W, Wang JJ. Long-term incidence of age-related macular degeneration. The Blue Mountain Eye Study. Arch Ophthalmology. 2007; 125:1089-1095.

30. Querques G, Massamba N, Srour M, Boulanger E, Georges A, Souied EH. Impact of reticular pseudodrusen on macular function. Retina. 2014;34:321-9.

31. Spaide RF. Age-related choroidal atrophy. Am J Ophthalmol. 2009;147:801-10.

32. Curcio CA, Messinger JD, Sloan KR, McGwin G, Medeiros NE, Spaide RF. Subretinal drusenoid deposits in non-neovascular agerelated macular degeneration: morphology, prevalence, topography, and biogenesis model. Retina. 2013;33:265-276.

33. Steinberg JS, Fleckenstein M, Holz FG, Schmitz-Valckenberg S. Foveal sparing of reticular drusen in eyes with early and intermediate age-related macular degeneration. Invest Ophthalmol Vis Sci. 2015;56:4267-74. 